\title{
Average Outage and Non-Outage Duration of Selective Decode-and-Forward Relaying
}

\author{
Nikola Zlatanov ${ }^{\dagger}$, Robert Schober ${ }^{\dagger}$, Zoran Hadzi-Velkov ${ }^{\dagger \dagger}$, and George Karagiannidis ${ }^{\dagger \dagger}$ \\ ${ }^{\dagger}$ The University of British Columbia, E-mail: \{zlatanov, rschober\}@ece.ubc.ca \\ ${ }^{\dagger \dagger}$ Ss. Cyril and Methodius University, Skopje, E-mail: zoranhv@feit.ukim.edu.mk \\ ${ }^{\dagger \dagger}$ Aristotle University Thessaloniki, E-mail: geokarag@auth.gr
}

\begin{abstract}
In this paper, assuming transmission with fixed rate and fixed power, we derive the average duration of capacity outage and non-outage events of selective decode-and-forward relaying with repetition coding over slow Rayleigh fading channels. Furthermore, we develop high signal-to-noise ratio (SNR) approximations for both durations which provide significant insight into the impact of various system and channel parameters. For high SNR, on a double logarithmic scale, both the average outage duration (AOD) and the average non-outage duration (ANOD) become straight lines when plotted as functions of the SNR. However, while the slope of the ANOD improves with increasing diversity order, the slope of the AOD is $-1 / 2$ independent of the diversity order.
\end{abstract}

\section{INTRODUCTION}

Cooperative diversity is an efficient technique to exploit the inherent spatial diversity of wireless networks [1]-[4]. In particular, opportunistic relaying, where only one out of the set of available relays is selected, extracts the full diversity of the channel and minimizes the loss in spectral efficiency caused by the repetition at the relays [1], [3]. For fixed rate transmission, the random nature of the propagation environment may lead to capacity outage events if the transmission rate exceeds the capacity of the end-to-end channel. Under these conditions, the performance of cooperative diversity systems is generally characterized by the outage probability. However, in systems with mobile nodes, the channel gains are slowly time varying and the outage events in neighboring coding blocks become correlated. This phenomenon is not captured by the outage probability itself but can be characterized by the average outage duration (AOD) and the average non-outage duration (ANOD). The AOD and ANOD are useful measures for the design of systems which exploit the temporal changes of the channel such as systems using automatic repeat request (ARQ) and multi-user scheduling.

In this paper, we derived the AOD and ANOD of a simple selective repetition coding (SRC) based decode-and-forward (DF) relaying protocol introduced in [1]. Besides the accurate results, we develop high signal-to-noise ratio (SNR) approximations which provide significant insight into the impact of the various system and channel parameters. Related work includes [5] and [6]. Compared to this paper, in [5], the direct link between the source and the destination was ignored and a different relay selection policy was considered. In [6], cooperative protocols with only one relay were studied. Furthermore, to the best of our knowledge, the ANOD and its asymptotic behavior have not been considered in any other work before.

Organization: In Section II, the system model and some definitions are presented. The AOD and ANOD for the considered SRC DF protocol are derived in Section III, and illustrated based on a numerical example in Section IV. Conclusions are drawn in Section V.

\section{PRELiminaries}

In this section, we present the adopted system and channel models as well as the definitions of the AOD and ANOD.

\section{A. System Model}

We consider a cooperative network consisting of a source $S$, a destination $D$, and $M$ relays denoted as $R_{i}, i \in\{1,2, \ldots, M\}$, operating in a Rayleigh fading enviroment. The channel gain of the direct link $(S \rightarrow D)$ is denoted by $X(t)$, the channel gain of the link between the source and relay $R_{i}\left(S \rightarrow R_{i}\right)$ is denoted by $Y_{i}(t)$, and the channel gain of the link between relay $R_{i}$ and the destination $\left(R_{i} \rightarrow D\right)$ is denoted by $Z_{i}(t)$. The average channel gain powers are defined as $E\left[X^{2}\right] \triangleq \Omega_{X}, E\left[Y_{i}^{2}\right] \triangleq \Omega_{Y}$, and $E\left[Z_{i}^{2}\right] \triangleq \Omega_{Z}$, where $E[\cdot]$ denotes expectation ${ }^{1}$. All nodes are assumed to transmit with equal power $P_{T}$, and $\Gamma_{0} \triangleq P_{T} / N_{0}$ denotes the transmit SNR, where $N_{0}$ is the power spectral density of the underlying additive white Gaussian noise.

We assume two-dimensional isotropic scattering around all nodes. Furthermore, $S, R_{i}, i \in\{1,2, \ldots, M\}$, and $D$ are assumed to be mobile, thus introducing maximum Doppler shifts $f_{m S}, f_{m R}$, and $f_{m D}$, respectively. Under these conditions, the channel gains $X(t), Y_{i}(t)$, and $Z_{i}(t)$ are time-correlated Rayleigh random processes and their auto-covariance functions and Doppler spectra are given in [7]. The time derivative of the channel gain $\alpha(t), \alpha \in\{X, Y, Z\}$, is denoted by $\dot{\alpha}(t)$. This derivative is independent of the channel gain itself and is a zero-mean Gaussian random variable (RV) with variance [8]

$$
\sigma_{\dot{\alpha}} \triangleq \pi f_{m \alpha} \sqrt{\Omega_{\alpha}}
$$

where $f_{m X} \triangleq \sqrt{f_{m S}^{2}+f_{m D}^{2}}, f_{m Y} \triangleq \sqrt{f_{m S}^{2}+f_{m R}^{2}}$, and $f_{m Z} \triangleq \sqrt{f_{m R}^{2}+f_{m D}^{2}}$.

\section{B. Relay Protocol}

The considered relaying protocol was introduced in [1]. The transmission is performed in two phases. In the first phase, the source broadcasts a codeword to an a priori selected best relay and to the destination. The relay decodes the codeword and forwards it to the destination in the second phase using repetition coding. The destination coherently combines the signals received in both phases and attempts to decode the transmitted message.

The single best relay for this SRC DF protocol is chosen as follows. Assume that for a relay to decode successfully its source-relay channel, $Y_{i}$, has to exceed some threshold $Y_{0}$. We collect the indices $i$ of all relays $R_{i}, i \in\{1,2, \ldots, M\}$, with $Y_{i}>Y_{0}$ in set $\mathcal{D}(t)$. The selected best relay, $R_{j}$, has the maximum relay-destination channel gain among all relays with indices in $\mathcal{D}(t)$, i.e., $Z_{j}(t)=\max \left\{Z_{i}(t)\right\}_{i \in \mathcal{D}(t)}$. We note

\footnotetext{
${ }^{1}$ Throughout this paper we omit reference to time $t$ whenever possible without giving rise to ambiguity.
} 
that since the relay-destination gains $Z_{i}(t)$ are independent, identically distributed (i.i.d.) Rayleigh RVs, the probability density function (PDF) of the gain of the best relay, $Z_{j}(t)$, depends only on the cardinality $k \triangleq|\mathcal{D}(t)|$ of set $\mathcal{D}(t)$. Hence, assuming $\mathcal{D}(t)$ contains $k$ elements, the PDF of $Z_{j}(t)$ is given by [9]

$$
f_{Z_{j}, k}(z)=k\left[1-\exp \left(-\frac{z^{2}}{\Omega_{Z}}\right)\right]^{k-1} \frac{2 z}{\Omega_{Z}} \exp \left(-\frac{z^{2}}{\Omega_{Z}}\right) .
$$

The instantaneous SNR of the end-to-end channel can be expressed as $\gamma(t)=\Gamma_{0} G^{2}(t)$, where $G(t)$ is given by

$$
G(t)=\left\{\begin{array}{cl}
\sqrt{X^{2}(t)+Z_{j}^{2}(t)} & \text { if } \mathcal{D}(t) \neq \emptyset \\
X(t) & \text { if } \mathcal{D}(t)=\emptyset
\end{array}\right.
$$

\section{Capacity Outage and Non-Outage Durations}

We assume the source transmits at a fixed rate of $R_{0} \mathrm{~b} / \mathrm{s} / \mathrm{Hz}$. Hence, the relays can decode if $\log _{2}\left(1+\Gamma_{0} Y_{i}^{2}(t)\right) / 2 \geq R_{0}$ and the decoding threshold at the relays is $Y_{0} \triangleq \sqrt{\left(2^{2 R_{0}}-1\right) / \Gamma_{0}}$. The mutual information of the cooperative end-to-end $S-D$ channel is given by [10]

$$
I(t)=\frac{1}{2} \log _{2}\left(1+\Gamma_{0} G^{2}(t)\right),
$$

and reliable decoding at the destination is possible as long as $I(t) \geq R_{0}$. On the other hand, decoding will fail (outage event) if $I(t)<R_{0}$ or equivalently

$$
G(t)<G_{0}
$$

where $G_{0} \triangleq \sqrt{\left(2^{2 R_{0}}-1\right) / \Gamma_{0}}=Y_{0}{ }^{2}$ Hence, the outage probability of the system is given by

$$
P_{\text {out }}=P\left(I(t)<R_{0}\right)=P\left(G(t)<G_{0}\right),
$$

where $P(A)$ denotes the probability of event $A$. We emphasize that for (6) to bear relevance for a practical system two conditions have to be satisfied [10], [11]. First, a coding block has to comprise a sufficiently large number of symbols such that capacity approaching codes exist. Second, the duration of a coding block, $T$, has to be sufficiently short such that the channel $G(t)$ is practically constant during the transmission of one codeword, i.e., $T$ has to be much smaller than the coherence time, $T_{\text {coh }}$, of the channel.

Although $G(t)$ is approximately constant within one coding block, it changes slowly from one coding block to the next. Thus, both the average duration of a capacity outage event (AOD) and the average duration of a capacity non-outage event (ANOD) are of interest for system design. Because of the relation between $I(t)$ and $G(t)$, the AOD is identical to the average duration that the random process $G(t)$ remains below $G_{0}$ after crossing that level in the downward direction. Thus, the AOD can be obtained as [12]

$$
O_{I}\left(R_{0}\right)=O_{G}\left(G_{0}\right)=P\left(G<G_{0}\right) / L_{G}\left(G_{0}\right),
$$

where $L_{G}\left(G_{0}\right)$ denotes the level crossing rate (LCR) of random process $G(t)$ at threshold $G_{0}$. Similarly, the ANOD is identical to the average duration that the random process $G(t)$ remains above $G_{0}$, after crossing that level in the upward direction. Thus, the ANOD is given by

$$
N_{I}\left(R_{0}\right)=N_{G}\left(G_{0}\right)=\left(1-P\left\{G<G_{0}\right\}\right) / L_{G}\left(G_{0}\right) .
$$

${ }^{2}$ Although $G_{0}=Y_{0}$ holds, it is convenient to keep both variables in the following since they indicate the considered link (source-relay link vs. end-toend link).
Based on (7) and (8) we can express the outage probability in terms of the AOD and the ANOD

$$
P\left(I<R_{0}\right)=P\left(G<G_{0}\right)=\frac{O_{G}\left(G_{0}\right)}{O_{G}\left(G_{0}\right)+N_{G}\left(G_{0}\right)} .
$$

In a practical system, the minimum duration of an outage event is equal to the duration of one coding block. In general, on average approximately $\left\lfloor O_{I}\left(R_{0}\right) / T\right\rfloor$ and $\left\lfloor N_{I}\left(R_{0}\right) / T\right\rfloor$ consecutive codewords will be decoded unsuccessfully and successfully, respectively. As a result, for example, in ARQ systems, the interval between two retransmission attempts should be chosen on the order of $O_{I}\left(R_{0}\right)$.

\section{AOD AND ANOD}

In this section, the exact and asymptotic expressions for the AOD and ANOD of the considered SRC cooperative protocol are derived. As can be observed from (7) and (8), the AOD and ANOD can be obtained easily if the LCR, $L_{G}\left(G_{0}\right)$, and the outage probability, $P\left(G \leq G_{0}\right)$, are known.

\section{A. LCR - Smooth Part of $G(t)$}

Random process $G(t)$ as a function of time $t$ is a function with discontinuities, i.e, there are points at which $\lim _{\epsilon \rightarrow 0} \mid G(t-$ $\epsilon)-G(t+\epsilon) \mid>0$. Thus, $G(t)$ is not differentiable at every point and the well-known Rice's formula [12] cannot be directly applied for obtaining the LCR. To circumvent this problem, we decompose the level crossings of $G(t)$ into two types. The first type corresponds to the time period when the cardinality $k$ of $\mathcal{D}(t)$ is fixed. During this time period, $G(t)$ is a smooth function. The second type of crossings is caused by changes in set $\mathcal{D}(t)$.

We first consider the level crossings that are caused by the smooth part of $G(t)$. To this end, it is convenient to denote the end-to-end gain for a set $\mathcal{D}(t)$ of cardinality $k$ by $G_{k}(t)$. Thus, by the total probability theorem the joint PDF of $G(t)$ and its derivative, $\dot{G}(t)$, can be expressed as

$$
f_{G \dot{G}}(g, \dot{g})=\sum_{k=0}^{M} f_{G_{k} \dot{G}_{k}}(g, \dot{g}) P(|\mathcal{D}|=k)
$$

where $f_{G_{k} \dot{G}_{k}}(g, \dot{g})$ is the joint PDF of $G_{k}(t)$ and its derivative, $\dot{G}_{k}(t)$, and

$$
P(|\mathcal{D}|=k)=\left(\begin{array}{c}
M \\
k
\end{array}\right) P\left(Y \geq Y_{0}\right)^{k} P\left(Y<Y_{0}\right)^{M-k}
$$

with $P\left(Y \geq Y_{0}\right)=1-P\left(Y<Y_{0}\right)$ and $P\left(Y<Y_{0}\right)=$ $1-\exp \left(-\bar{Y}_{0}^{2} / \Omega_{Y}\right)$. Since for a given $k, G(t)$ is a smooth function, we can apply Rice's formula [12]

$$
L_{G_{S}}\left(G_{0}\right)=\int_{0}^{\infty} \dot{g} f_{G \dot{G}}\left(G_{0}, \dot{g}\right) d \dot{g}
$$

to obtain the corresponding LCR,

$$
L_{G_{S}}\left(G_{0}\right)=\sum_{k=0}^{M} L_{G_{k}}\left(G_{0}\right) P(|\mathcal{D}|=k),
$$

where $L_{G_{k}}\left(G_{0}\right)$ denotes the LCR of $G_{k}$,

$$
L_{G_{k}}\left(G_{0}\right)=\int_{0}^{\infty} \dot{g} f_{G_{k} \dot{G}_{k}}\left(G_{0}, \dot{g}\right) d \dot{g} .
$$

For a given cardinality $k, Z_{j}(t)$ and its derivative $\dot{Z}_{j}(t)$ are independent and $\dot{Z}_{j}(t)$ is a zero mean Gaussian RV with 
variance $\sigma_{\dot{Z}}^{2}$ [13]. Thus, exploiting (2) and following the method in [14], we obtain the LCR of $G_{k}$ as

$$
\begin{gathered}
L_{G_{k}}\left(G_{0}\right)=\sqrt{\frac{2}{\pi}} \frac{2 k}{\Omega_{X} \Omega_{Z}} \int_{0}^{G_{0}} \sqrt{\left(G_{0}^{2}-z^{2}\right) \sigma_{\dot{X}}^{2}+z^{2} \sigma_{\dot{Z}}^{2}} \\
\exp \left(-\frac{G_{0}^{2}-z^{2}}{\Omega_{X}}\right)\left[1-\exp \left(-\frac{z^{2}}{\Omega_{Z}}\right)\right]^{k-1} z \exp \left(-\frac{z^{2}}{\Omega_{Z}}\right) d z,
\end{gathered}
$$

for $k>0$. For $k=0, L_{G_{k}}\left(G_{0}\right)$ is equal to the LCR of $X(t)$ which is given by [13]

$$
L_{\alpha}\left(G_{0}\right)=\frac{\sigma_{\dot{\alpha}}}{\sqrt{2 \pi}} \frac{2 G_{0}}{\Omega_{\alpha}} \exp \left(-\frac{G_{0}^{2}}{\Omega_{\alpha}}\right), \quad \alpha \in\{X, Y, Z\} .
$$

The LCR of the smooth part of $G(t)$ can be obtained by combining (13)-(16).

\section{B. LCR - Discontinuities of $G(t)$}

Let us define $t_{-} \triangleq \lim _{\epsilon \rightarrow 0}(t-\epsilon)$ and $t_{+} \triangleq \lim _{\epsilon \rightarrow 0}(t+\epsilon)$. A discontinuity of $G(t)$ at time $t$ can happen if and only if a relay was removed from or added to set $\mathcal{D}(t)$, i.e., $\mathcal{D}\left(t_{-}\right) \neq \mathcal{D}\left(t_{+}\right)$. In particular, upward crossings of $G_{0}$ can happen only if a relay is added to $\mathcal{D}(t)$. Thus, denoting the cardinality of the set at time $t_{-}$by $k-1$, we have $k-1=\left|\mathcal{D}\left(t_{-}\right)\right|<\left|\mathcal{D}\left(t_{+}\right)\right|=k$. A upward level crossing happens if we have for the corresponding channel gains

$$
G_{k-1}\left(t_{-}\right)<G_{0} \text { AND } G_{k}\left(t_{+}\right) \geq G_{0} .
$$

For computation of the LCR, we first count the average number of changes of the cardinality from $k-1$ to $k$. The fraction of these changes that lead to level crossings is obtained by a simple multiplication with the probability of the event in (17). A subsequent summation over all $k \in\{1,2, \ldots M\}$ yields the LCR due to discontinuities of $G(t)$.

A new relay is added to $\mathcal{D}(t)$ if the source-relay gain, $Y_{i}(t)$, of relay $R_{i}$ that is not included in $\mathcal{D}(t)$, yet, crosses $Y_{0}$. Let us put all $k-1$ source-relay gains, $Y_{i}(t)$, with $Y_{i}(t) \geq Y_{0}$ into set $\mathcal{A}$ and all $M-k+1$ remaining source-relay gains into set $\overline{\mathcal{A}}$. $|\mathcal{D}(t)|$ will change from $k-1$ to $k$ if one of the elements of $\overline{\mathcal{A}}$ crosses the threshold $Y_{0}$ in an upward direction. Let the element of $\overline{\mathcal{A}}$, out of the $M-k+1$ possible elements, that crosses $Y_{0}$ in an upward direction be $Y_{i}$. Furthermore, let $\overline{\mathcal{A}}_{i}$ denote a set equal to the set $\overline{\mathcal{A}}$ but with $Y_{i}$ removed, i.e., $\overline{\mathcal{A}}_{i}=\overline{\mathcal{A}} \backslash Y_{i}$. The average number of upward crossings of $Y_{i}$ is $L_{Y}\left(Y_{0}\right)$, cf. (16), and the average number of upward crossings made by $Y_{i}$ when the elements of $\overline{\mathcal{A}}_{i}$ are smaller than $Y_{0}$ and the elements of $\mathcal{A}$ are larger than $Y_{0}$ is

$$
\begin{aligned}
& L_{Y}\left(Y_{0}\right) \prod_{Y_{k} \in \mathcal{A}} P\left(Y_{k} \geq Y_{0}\right) \prod_{Y_{l} \in \overline{\mathcal{A}}_{i}} P\left(Y_{l}<Y_{0}\right) \\
& =L_{Y}\left(Y_{0}\right) P\left(Y \geq Y_{0}\right)^{k-1} P\left(Y<Y_{0}\right)^{M-k} .
\end{aligned}
$$

Since the two events that (a) $Y_{i}$ crosses $Y_{0}$ when $Y_{j} \in \overline{\mathcal{A}}_{i}$ and (b) $Y_{j}(t)$ crosses $Y_{0}$ when $Y_{i} \in \overline{\mathcal{A}}_{j}, i \neq j$, are mutually exclusive, the total number of upward crossings made by all elements of $\overline{\mathcal{A}}$ is the sum of the crossings made by the individual elements of $\overline{\mathcal{A}}$ given by

$$
\begin{aligned}
& \sum_{Y_{i} \in \overline{\mathcal{A}}} L_{Y}\left(Y_{0}\right) P\left(Y \geq Y_{0}\right)^{k-1} P\left(Y<Y_{0}\right)^{M-k} \\
& =(M-k+1) L_{Y}\left(Y_{0}\right) P\left(Y \geq Y_{0}\right)^{k-1} P\left(Y<Y_{0}\right)^{M-k} .
\end{aligned}
$$

Eq. (19) multiplied with the probability of event (17) gives the average number of upward crossings of $G_{0}$ due to the discontinuities of $G(t)$ for one specific $\overline{\mathcal{A}}$. Since $\overline{\mathcal{A}}$ contains $M-k+1$ of the $M$ gains $Y_{i}(t), i \in\{1,2, \ldots, M\}$, there are $\left(\begin{array}{c}M \\ M-k+1\end{array}\right)$ different sets $\overline{\mathcal{A}}$. Hence, using $(M-k+1)\left(\begin{array}{c}M \\ M-k+1\end{array}\right)=$ $k\left(\begin{array}{c}M \\ k\end{array}\right)$ and summing over $k \in\{1,2, \ldots, M\}$, based on (19), the LCR caused by the discontinuities of $G(t), L_{G_{D}}\left(G_{0}\right)$, is obtained as

$$
\begin{aligned}
& L_{G_{D}}\left(G_{0}\right)=L_{Y}\left(Y_{0}\right) \sum_{k=1}^{M}\left[k\left(\begin{array}{c}
M \\
k
\end{array}\right) P\left(Y \geq Y_{0}\right)^{k-1}\right. \\
& \left.\quad \times P\left(Y<Y_{0}\right)^{M-k} P\left(G_{k-1}<G_{0} \text { AND } G_{k} \geq G_{0}\right)\right] .
\end{aligned}
$$

For the term $P\left(G_{k-1}<G_{0}\right.$ AND $\left.G_{k} \geq G_{0}\right)$, we obtain for $k=1$

$$
\begin{aligned}
P\left(G_{k-1}\right. & \left.<G_{0} \text { AND } G_{k} \geq G_{0}\right) \\
= & \frac{\Omega_{Z}}{\left(\Omega_{X}-\Omega_{Z}\right)}\left[\exp \left(-\frac{G_{0}^{2}}{\Omega_{X}}\right)-\exp \left(-\frac{G_{0}^{2}}{\Omega_{Z}}\right)\right]
\end{aligned}
$$

and for $k>1$

$$
\begin{aligned}
P\left(G_{k-1}\right. & \left.<G_{0} \text { AND } G_{k} \geq G_{0}\right) \\
= & \frac{(k-1) e^{-G_{0}^{2} / \Omega_{Z}}}{\left(\Omega_{X}-\Omega_{Z}\right)} \int_{0}^{G_{0}^{2}}\left(1-e^{-z / \Omega_{Z}}\right)^{k-2} e^{-z / \Omega_{Z}} \\
& \times\left[\exp \left(-\left(G_{0}^{2}-z\right) \frac{\Omega_{Z}-\Omega_{X}}{\Omega_{X} \Omega_{Z}}\right)-1\right] d z .
\end{aligned}
$$

\section{Exact $A O D$ and $A N O D$}

As mentioned before, for computation of both the AOD and ANOD, we require the LCR and the outage probability. Based on the results of the previous two subsections, the LCR, $L_{G}\left(G_{0}\right)$, of random process $G(t)$ is obtained as

$$
L_{G}\left(G_{0}\right)=L_{G_{S}}\left(G_{0}\right)+L_{G_{D}}\left(G_{0}\right),
$$

where we have added the crossings due to the smooth and discontinuous parts of $G(t)$.

The outage probability of $G(t)$ is obtained as

$$
P\left(G(t)<G_{0}\right)=\sum_{k=0}^{M} P\left(G_{k}(t)<G_{0}\right) P(|\mathcal{D}|=k),
$$

where for $k=0, P\left(G_{0}(t)<G_{0}\right)=P\left(X(t)<G_{0}\right)=1-$ $\exp \left(-G_{0}^{2} / \Omega_{X}\right)$, and for $k>0$,

$$
P\left(G_{k}(t)<G_{0}\right)=\int_{0}^{G_{0}^{2}}\left[1-\exp \left(-\frac{G_{0}^{2}-x}{\Omega_{Z}}\right)\right]^{k} \frac{e^{-x / \Omega_{X}}}{\Omega_{X}} d x
$$

Finally, the AOD and ANOD are obtained by inserting (23)(25) in (7) and (8), respectively. We note that all remaining integrals can be solved in closed form by using the binomial expansion. This is omitted here for brevity.

\section{Asymptotic AOD and ANOD}

To gain more insight, it is interesting to consider the high SNR regime, i.e., $\Gamma_{0} \rightarrow \infty$, or equivalently, $G_{0} \rightarrow 0$ and $Y_{0} \rightarrow 0$. The results in this section were obtained by exploiting the expansions $e^{-t} \sim 1-t$ and $\Gamma(k, t) \sim \Gamma(k)-t^{k} / k$ as $t \rightarrow 0$ for the exponential and incomplete Gamma functions [15], respectively. Here, the notation $g(x) \sim h(x)$ as $x \rightarrow x_{0}$ means that $g(x)$ and $h(x)$ are asymptotically equivalent, i.e., $\lim _{x \rightarrow x_{0}} g(x) / h(x)=1$. 
Applying the aforementioned expansions, for high SNR, we obtain from (23) for the asymptotic LCR

$$
\begin{aligned}
L_{G}\left(G_{0}\right) & \sim \sqrt{\frac{2}{\pi}} \frac{{\sqrt{2^{2 R_{0}}-1}}^{2 M+1}}{\Omega_{X} \Omega_{Y}^{M} \Gamma_{0}^{M+1 / 2}}\left(\sigma_{\dot{X}}+\sum_{k=1}^{M}\left(\begin{array}{c}
M \\
k
\end{array}\right)\left(\frac{\Omega_{Y}}{\Omega_{Z}}\right)^{k}\right. \\
\times & {\left.\left[\sigma_{\dot{X} 2} F_{1}\left(-\frac{1}{2}, k ; k+1 ; 1-\frac{\sigma_{\dot{Z}}^{2}}{\sigma_{\dot{X}}^{2}}\right)+\sigma_{\dot{Y}} \frac{\Omega_{Z}}{\Omega_{Y}}\right]\right), \quad \text { (26) } }
\end{aligned}
$$

where ${ }_{2} F_{1}(\cdot, \cdot ; \cdot ; \cdot)$ denotes the Gauss' hypergeometric function $[15,(15.1)]$. Similarly, we obtain for the asymptotic outage probability

$$
P\left(G<G_{0}\right) \sim \frac{\left(2^{2 R_{0}}-1\right)^{M+1}}{\Omega_{X} \Omega_{Y}^{M} \Gamma_{0}^{M+1}} \sum_{k=0}^{M}\left(\begin{array}{c}
M \\
k
\end{array}\right) \frac{1}{k+1}\left(\frac{\Omega_{Y}}{\Omega_{Z}}\right)^{k} .
$$

The asymptotic AOD and ANOD are obtained by inserting (26), (27) in (7) and (8), respectively.

To gain more insight, we consider the case of a symmetric network with $\Omega_{X}=\Omega_{Y}=\Omega_{Z}=\Omega$ and $\sigma_{\dot{X}}=\sigma_{\dot{Y}}=\sigma_{\dot{Z}}=$ $\pi f_{m} \sqrt{\Omega}$. In this case, we obtain for the AOD

$$
O_{G} \sim \frac{\sqrt{2^{2 R_{0}}-1}}{\sqrt{2 \pi} f_{m}(M+1) \sqrt{\Gamma_{0}}}
$$

and for the ANOD

$$
N_{G} \sim \frac{\Omega^{M+1 / 2} \Gamma_{0}^{M+1 / 2}}{\sqrt{2 \pi} f_{m}\left(2^{M+1}-1\right){\sqrt{2^{2 R_{0}}-1}}^{2 M+1}} .
$$

From (28) and (29) we observe that for high SNR, on a doublelogarithmic scale, the AOD and ANOD decrease and increase linearly with the SNR, respectively. However, while the slope of the AOD is equal to $-1 / 2$ independent of $M$, the slope of the ANOD is equal to $M+1 / 2$ and thus, similar to the outage probability, strongly benefits from an increasing number of relays. Hence, while the number of available relays has a relatively minor effect on the average number of successive codewords affected by an outage, the effect on the average number of successive correctly decoded codewords is major. This will be illustrated by an example in the next section.

\section{NUMERICAL EXAMPLE}

In Fig. 1, we show the AOD and ANOD vs. SNR, $\Gamma_{0}$, for $R_{0}=1 \mathrm{~b} / \mathrm{s} / \mathrm{Hz}, \Omega_{X}=1.3, \Omega_{Y}=0.9, \Omega_{Z}=0.8$, and $f_{m X}=$ $f_{m Y}=f_{m Z}=50 \mathrm{~Hz}$. The exact analytical results from Section III-C are confirmed by simulation results and the high SNR approximations from Section III-D become tight for $\Gamma_{0}>18$ $\mathrm{dB}$. The fundamentally different impact of the number of relays on the AOD and ANOD is confirmed by Fig. 1. For example, assuming an SNR of $20 \mathrm{~dB}$ and a codeword duration of $T=$ $10^{-5} \mathrm{~s}$, for $M=1,3$, and 7 on average $O_{I}\left(G_{0}\right) / T=67,35$, and 18 consecutive codewords are in outage. In contrast, for the same numbers of relays on average $N_{I}\left(G_{0}\right) / T=5.8 \times 10^{4}$, $1.07 \times 10^{7}$, and $4.7 \times 10^{11}$ consecutive codewords are not in outage.

\section{Conclusions}

In this paper, we derived the AOD and ANOD of an SRC DF cooperative diversity system. Besides the exact expressions for the AOD and ANOD, we developed insightful high SNR approximations. This asymptotic analysis revealed that while increasing the number of relays has a moderate positive effect on the AOD, it has a much larger positive effect on the ANOD.

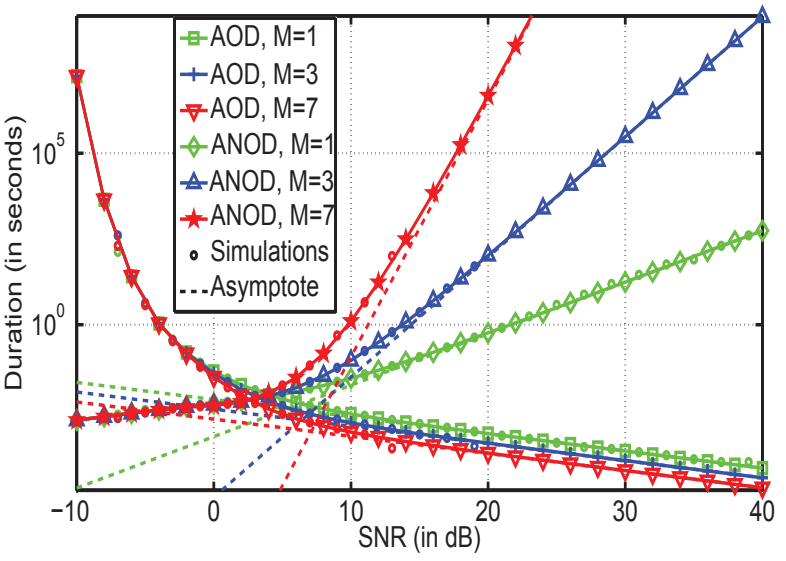

Fig. 1. AOD and ANOD vs. SNR, $\Gamma_{0}$. Solid lines with markers: Exact results, cf. Section III-C. Dashed lines: Asymptotic results, cf. Section III-D. Circle markers: Simulations.

In a practical system, the AOD may be used as a design guideline for the minimum interval between two retransmission attempts in an ARQ system or the duration of a scheduling slot in a multi-user system. The ANOD on the other hand can serve as an estimate for the duration of interruption free transmission.

\section{REFERENCES}

[1] E. Beres, R. Adve, "Selection Cooperation in Multi-Source Cooperative Networks", IEEE Trans. Wireless Comm., vol. 7, pp. 118-127, Jan. 2008.

[2] A. Bletsas, A. Khisti, D. P. Reed, and A. Lippman, "A Simple Cooperative Diversity Method Based on Network Path Selection", IEEE J. Selec. Areas Comm., vol. 24, pp. 659-672, Mar. 2006.

[3] A. Nosratinia and T. E. Hunter, "Grouping and Partner Selection in Cooperative Wireless Networks", IEEE J. Selec. Areas Comm., vol. 54, pp. 369-378, Feb. 2006.

[4] J. N. Laneman and G. W. Wornell, "Distributed Space Time-Coded Protocols for Exploiting Cooperative Diversity in Wireless Networks", IEEE Trans. Inf. Theory, vol. 49, pp. 2415-2425, Oct. 2003.

[5] Zoran Hadzi-Velkov, Nikola Zlatanov, "Outage Rates and Outage Durations of Opportunistic Relaying Systems", IEEE Comm. Lett. vol.14, pp. 148150, Feb. 2010.

[6] Nikola Zlatanov, Zoran Hadzi-Velkov, George K. Karagiannidis, and Robert Schober, "Outage Rate and Outage Duration of Decode-and-Forward Cooperative Diversity Systems", accepted for presentation at IEEE International Conf. Commun. (ICC), Jun. 2011. Online: http: //www. ece.ubc.ca/ $\sim$ zlatanov/pdf/icc2011.pdf

[7] A. S. Akki and F. Haber, "A Statistical Model of Mobile-to-Mobile Land Communication Channel" IEEE Trans. Veh. Technol., vol. 35, pp. 2-7, Feb. 1986.

[8] A. S. Akki, "Statistical Properties of Mobile-to-Mobile Land Communication Channel",IEEE Trans. Veh. Technol., vol. 43, pp. 826-831, Nov. 1994.

[9] H. A. David, H. N. Nagaraja, "Order Statistics", Wiley, 3rd Edition 2003.

[10] D. Tse and P. Viswanath, "Fundamentals of Wireless Communications", Cambridge Univ. Press, 2005.

[11] L. H. Ozarow, S. Shamai (Shitz), and A. D. Wyner, "Information Theoretic Considerations for Cellular Mobile Ratio", IEEE Trans. Veh. Technol., vol. 43, pp. 359-378, May 1994.

[12] S. O. Rice, "Statistical Properties of a Sine Wave Plus Noise", Bell System Tech. J., vol. 27, pp. 109-157, Jan. 1948.

[13] X. Dong, N.C. Beaulieu, "Average Level Crossing Rate and Average Fade Duration of Selection Diversity", IEEE Comm. Lett., vol. 5, pp. 396-398, Aug. 2002.

[14] C.D. Iskander, P. T. Mathiopoulos, ”Analytical Level Crossing Rates and Average Fade Durations for Diversity Techniques in Nakagami Fading Channels", IEEE Trans. Comm., vol. 50 , pp. 1301-1309, Aug. 2002.

[15] M. Abramowitz and I. A. Stegun, "Handbook of Mathematical Functions with Formulas, Graphs, and Mathematical Tables", 9th ed. New York: Dover, 1970 